Research Paper

\title{
A real-world 1:1 propensity-matched study revealed unmarried status was independently associated with worse survival for patients with renal clear cell carcinoma
}

\author{
Shi-Long Zhang ${ }^{1 *}$, Hai-Tao Sun ${ }^{2,3^{*}}$, Zhan-Ming Li ${ }^{1}$, Zheng-Yan Zhang ${ }^{1}$, Wen-Rong Wang ${ }^{4}$, Xin Wang ${ }^{5}$, \\ Zhi-Ming Wang, ${ }^{6,}$, Li-Shun Wang ${ }^{\circledR}$ \\ 1. Minhang Hospital, Fudan University, Shanghai 201199, China; \\ 2. Institute of Fudan-Minhang Academic Health System, Minhang Hospital, Fudan University, Shanghai 201199, China: \\ 3. Department of Interventional Radiology, Zhongshan Hospital, Fudan University, Shanghai 200032, P.R. China; \\ 4. Faculty of Physical Education, Shandong Normal University, Jinan 250014, P.R. China; \\ 5. Department of acupuncture and moxibustion, Central Hospital of Shanghai Xuhui District, Shanghai 200031, P.R. China; \\ 6. Department of Medical oncology, Zhongshan Hospital, Fudan University, No. 180 Fenglin Road, Shanghai, 200032, P.R. China; \\ 7. Xiamen branch, Zhongshan Hospital, Fudan University, No. 668 Jinhu Road, Xiamen, 361000, P.R China. \\ *Shi-Long Zhang and Hai-Tao Sun contribute equally to this paper.
}

$\square$ Corresponding authors: Li-Shun Wang, M.D., Ph.D., Institute of Fudan-Minhang Academic Health System, Minhang Branch, Zhongshan hospital, Fudan University, No. 170 Xinsong Road, Minhang, Shanghai, 200032, P.R. China. Tel: 86-021-60267405; E-mail: lishunwang@fudan.edu.cn. Zhi-Ming Wang, M.D., Ph.D., Department of Medical oncology, Zhongshan Hospital, Fudan University, No. 180 Fenglin Road, Shanghai, 200032, P.R. China; Xiamen branch, Zhongshan Hospital, Fudan University, No. 668 Jinhu Road, Xiamen, 361000, P.R China. Tel: 86-021-64041990; E-mail: wzming@126.com

(1) Ivyspring International Publisher. This is an open access article distributed under the terms of the Creative Commons Attribution (CC BY-NC) license (https://creativecommons.org/licenses/by-nc/4.0/). See http://ivyspring.com/terms for full terms and conditions.

Received: 2018.11.23; Accepted: 2019.05.07; Published: 2019.06.09

\begin{abstract}
Background: Marital status has been reported as an independent prognostic factor for survival in various cancers, but it has been rarely studied in renal clear cell carcinoma (ccRCC). In this study, we aimed to assess the impact of marital status on the survival of ccRCC patients.

Methods: We retrospectively investigated the Surveillance, Epidemiology, and End Results (SEER) database and identified 68599 of ccRCC patients between 1973 and 2015. These patients were divided into married, single, divorced and widowed groups. The survival differences among these groups were assessed by Kaplan-Meier method and log-rank test. Multivariate Cox regression analyses were performed to identify the overall survival (OS) and cancer-specific survival (CSS) independent factors. Furthermore, 1:1 propensity score matching (PSM) analysis was performed to minimize the potential confounding factors.

Results: Of the 68599 ccRCC patients, 44553 (64.95\%) patients were married, 7410 (10.80\%) were divorced, 10663 (15.54\%) were single, and 5973 (8.71\%) were widowed. The 5 -year OS was $79.0 \%, 73.8 \%, 77.3 \%$, and $66.4 \%$ in the married, divorced, single, and widowed groups, respectively $(p=0.001)$ and the corresponding 5 -year CSS rates were $85.5 \%, 83.3 \%, 80.8 \%, 76.5 \%$, respectively. Multivariate Cox regression analysis marital status was the independent prognostic factor for OS and CSS. Compared with the married patients, the divorced, single, and widowed patients faced increased higher mortality risks for OS and CSS. In stratified analyses by sex, surgery conditions and cancer stages, those unmarried patients still had worse prognosis. The results were further confirmed in the 1: 1 matched group.

Conclusion: Unmarried ccRCC patients experienced worse survival than their married counterparts. Among the unmarried patients, the widowed suffered the highest mortality risks for OS and CSS.
\end{abstract}

Key words: Renal clear cell carcinoma; marital status; cancer survival; propensity score matching; SEER

\section{Introduction}

Renal cell carcinoma (RCC) is a genitourinary malignancy. Its incidence has significantly increased ${ }^{1}$,

and contributed to approximately 63,990 new cases and approximately 693,000 deaths worldwide in 
20172. Among the histological subtypes of RCC, clear cell RCC (ccRCC) is the most common subtype, responsible for $75-80 \%$ of all RCC cases. The prognosis of ccRCC is determined by many factors, including age, sex, disease stage, Fuhrman nuclear grade, tumor size, molecular pathogenesis and treatment strategies. Nowadays people have realized that an integrative concept of health and disease should include the interaction of biology, psychology and sociology, which is also known as biological-psychology-social medical model ${ }^{3-4}$.

Recently, results from considerable literature have disclosed that that married patients have superior survival compared to the unmarried in various cancers ${ }^{5}$, such as soft tissue sarcoma ${ }^{6}$, liver cancer $^{7}$ and colon cancer ${ }^{8}$. This interesting phenomenon arise much public attentions. It is postulated that married status could contribute to optimistic psychological, enough social support, decent incomes, healthy lifestyle and comfortable living conditions. Similarly, previous studies reported that that marital status was a prognostic factor of survival in kidney cancer patients ${ }^{9-11}$. However, all of them merely focus on all kinds of kidney cancer without differentiating pathological subtypes. As we all know, RCC is a highly heterogenous tumor, ccRCC and other subtypes (eg, papillary, chromophobe) have distinct pathologies and biological behaviors, or even different long-term survival. So conducting analysis on the survival of ccRCC and other subtypes separately might be more important and reasonable. Furthermore, previous studies had significant imbalance baseline that married people were more likely to be diagnosed at an earlier stage and receive surgery compared to the unmarried. Thus, the impact of marital status on the survival of ccRCC has not been rigorously investigated.

In this study, we conducted a comprehensive study that involved a large sample ccRCC patients diagnosed between 1973 and 2015 to explore the relationship between marital status and ccRCC survival, as well as the potential underlying mechanisms. Furthermore, we also conducted 1:1 propensity score matching (PSM) analysis, a powerful method to minimize selection bias, to created $1: 1$ matched cohort with well-balanced baseline characteristics. In addition, we performed Cox proportional hazards regression to explore the impact of marital status on ccRCC patients in the matched cohort.

\section{Patients and Methods}

\section{Patient selection}

Our study used the SEER database-18 cohort database [Incidence -SEER 18 Regs Research Data +
Hurricane Katrina Impacted Louisiana Cases, Nov 2017 Sub (1973-2015 varying)], released in November 2017, as data source ${ }^{12}$. We got access to the SEER database with the ID number 13264-Nov2017. Using the SEER-stat software (SEER*Stat 8.2.1), patients with ICD-O-3 (International Classification of Diseases for Oncology, 3rd edition) site code C64.9 diagnosed between 1973 and 2015 were identified from the SEER database. Patients were included according to the following criteria: (1) their ICD-O-3 morphology code indicated ccRCC; (2) they aged more than 18 years at diagnosis; (3) their marital status were known; (4) they were diagnosed with ccRCC only or more than one primary cancer but ccRCC was the first; (5) their cause of death was known; (6) their survival time were known and greater than 0 month.

\section{Study variables}

The sex, age, race, diagnosis year, pathological grade, marital status, AJCC stage, surgery status, median household income, insurance status, cause of death, vital status and survival time from the SEER database. Marital status was categorized as married, divorced, single and widowed. Additionally, marital status was also categorized as married and unmarried (single, divorced and widowed) groups in the 1:1 PSM analysis. Patients were divided into three groups: 18 to 49 years, 50 to 59 years, 60 to 69 years, 70 to 79 years, and $\geq 80$ years. Race was classified as white, black, or others (American Indian/AK Native, Asian/Pacific Islander). Diagnosis years were divided into three periods (1973-1993,1994-2004, and 2005-2015) to eliminate the survival benefits brought by targeted therapy in recent years. County-level median household income was included to represent patients' socioeconomic status and stratified into quintiles for analysis: Quartile 1 (<US \$48700), Quartile 2 (US \$48701-56200), Quartile 3 (US \$56201-66931), and Quartile 4 (>US \$66931). The included patients were furthermore stratified into "insured", "uninsured" and "unknown" groups according to their insurance status. Tumor stage were listed as stage I, stage II, stage III, stage IV according to the American Joint Committee on Cancer staging (AJCC sixth edition).

\section{Statistical analyses}

The demographic and clinical data were presented by percentage (\%). Differences in baseline characteristics were compared by $\chi^{2}$ test. The primary endpoints in our study, overall survival (OS) and cancer-specific survival (CSS), were calculated by Kaplan-Meier method and log-rank test was used to detect survival differences. Multivariate Cox regression analysis were performed to identify the independent prognostic factors for ccRCC patients. 
To further control for potential baseline confounding factors across groups, we carried out 1:1 PSM analysis based on marital status (married and unmarried group) to re-examine the impact of marital status. In our study, a 1:1 pair matching (without replacement) was conducted through nearest neighbor method with a caliper of 0.1 times the standard deviation of the propensity score $^{13}$. The matching was performed by the MatchIt package in $\mathrm{R}$ (version 3.5.1). Standardized differences (SD) were used to examine the balance across baseline covariates before and after matching, and a SD below 0.1 was reliable enough to provide well-balanced covariates after matching ${ }^{14}$.

All statistical analyses and figures were generated using the tableone, rms, survival, survminer, ggplot2 and MatchIt packages in $R$ (version 3.5.1), unless otherwise specified. All $p$ values were two-sided with statistical significance defined at $<0.05$.

\section{Results}

\section{Patient baseline characteristics}

A total of 68599 eligible patients diagnosed from 1973 to 2015 in the SEER database were included. 43271 (63.08\%) were male and 25328 (36.92\%) were female, $44553(64.95 \%)$ were married, $7410(10.80 \%)$ were divorced, 10663 (15.54\%) were single, and 5973 (8.71\%) widowed. Baseline characteristics of ccRCC patients according to marital status were listed in Table 1. Difference of baseline characteristics were noticed significantly in all subgroups. Especially, married group had the highest percentage of male patients $(69.2 \%)$ and white patients $(83.3 \%)$, while among unmarried patients, single group had the highest percentage of male patients $(63.3 \%)$ and widowed group had the highest percentage of female (72.6\%). Widowed patients tended to be in age groups of $60-69(25.8 \%), 70-79(39.3 \%)$, and $\geq 80$ years $(26.3 \%)$, while the single patients were predominantly in younger age group of $18-49(33.4 \%)$, and more tended to present with smaller tumor sizes $(54.7 \%)$ and early stages $(65.2 \%)$, compared to other unmarried groups.

\section{Impact of marital status on overall survival (OS)}

The OS of ccRCC patients was calculated by Kaplan-Meier method. The results showed a significant survival difference according to marital status (log-rank test $p<0.001$ ) (Figure 1A). The 5-year OS was $79.0 \%$ in the married group, $73.8 \%$ in the divorced group, $77.3 \%$ in the single group, and $66.4 \%$ in the widowed group $(p<0.001)$. Univariate analysis identified marital status, age, race, diagnosis year, histological type, pathological grade, tumor size, AJCC stage, surgery status, median household income and insurance status as significant factors associated with OS $(p<0.001)$. After controlling for above-mentioned factors, multivariate Cox regression analysis showed that, compared to the married (as the reference group), divorced (hazard ratio (HR), 1.33, 95\% confidence interval (CI): 1.27-1.40), single (HR, 1.26, 95\%CI: 1.20-1.32), and widowed (HR, 1.42, 95\% CI: 1.35-1.49) patients had higher death risks for OS (Table 2). In addition, widowed patients had the lowest rate and the highest death risks for OS. Notably, socioeconomic factors including median household income and insurance status were significantly associated with OS in both univariate and multivariate analysis.

\section{Impact of marital status on cancer-specific survival (CSS)}

The 5-year CSS rates for married patients, divorced, widowed, single patients were $85.5 \%$, $83.3 \%, 80.8 \%, 76.5 \%$, respectively $(p<0.001)$ (Figure 1B, Table 3). By univariate analysis, all the baseline characteristics were found to be associated with CSS among ccRCC patients. In addition, patients with insurance had better CSS than those without (5-year CSS rate $85.2 \%$ vs. $83.2 \%, p<0.001$ ). When the aforementioned covariates were adjusted in multivariate Cox regression analysis, marital status was confirmed as an independent prognostic factor, with worse CSS among unmarried patients (divorced, HR, 1.16, 95\% CI 1.08-1.25, $p<0.001$; single, HR, 1.09, 95\% CI 1.02-1.16, $p=0.006$; widowed, HR, 1.20, 95\% CI 1.13-1.28, $p<0.001$ ). However, multivariate Cox regression analysis did not support the association between insurance status and CSS (HR, 1.04, 95\% CI $0.96-1.146, p=0.314)$.

\section{Subgroup analysis stratified by sex, surgery, and cancer stage}

Multiple variables including have been identified as prognostic factors for ccRCC mortality, and those variables also been verified independently in our study. Hence, subsequently we divided all ccRCC patients into several subgroups stratified by those variables and investigated their impacts on CSS. The survival curves of the patients within sex, surgery and AJCC stage group were shown in Figure 2, Figure 3 and Figure 4, respectively. Marital status remained an independent prognostic factor in almost all subgroups $(p<0.001)$ (Table 4$)$. Furthermore, in the Cox regression analysis, we also observed several interesting findings: 1) The widowed patients suffered the worst CSS in each sex, surgery and AJCC stage subgroups, and being widowed was associated 
with the highest death risks compared to other unmarried status. 2) Being divorced would raise death risks in comparison to being married in males, patients with surgery, and all AJCC stages. 3) No significant survival differences were noticed between the married and single group in the subgroup of female, and stage II stage III.

\section{Survival analysis of $\operatorname{ccRCC}$ patients in the $1: 1$ matched cohort}

To eliminate influences of confounding factors across the baseline characteristics and ensure our observations were reliable and stable, we conducted a 1:1 matched cohort analysis through PSM method. After matching, we had 18028 ccRCC patients including 9014 married and another 9014 unmarried patients in our subsequent analysis. Distribution of the baseline characteristics was well-balanced in the matched cohort (Table 5). In general, an absolute SD < 0.10 indicated a negligible difference across the groups; and the largest SD was 0.03 in the matched cohort.

Even so, unmarried patients persisted to suffer more significant survival disadvantages than married patients in the Kaplan-Meier analysis. The 5-year OS rate in married patients was $75.8 \%$ while 5 -year OS rate was $68.3 \%$ in unmarried group $(p<0.001)$ (Figure $5 \mathrm{~A})$. Like OS, the 5 -year CSS rate was $84.4 \%$ in the married group and $80.7 \%$ in unmarried group $(p<$ 0.001) (Figure 5B). Despite the basically comparable variables across two groups, we furthermore performed univariate Cox regression to make a more accurate conclusion.

Table 1. Baseline characteristics of ccRCC patients according to marital status in the SEER database

\begin{tabular}{|c|c|c|c|c|c|c|}
\hline Characteristic & Total (\%) & Married (\%) & Divorced (\%) & Single (\%) & Widowed (\%) & \\
\hline & $68599(100)$ & $44553(64.95)$ & $7410(10.80)$ & $10663(15.54)$ & $5973(8.71)$ & $p$ \\
\hline Sex & & & & & & $<0.001$ \\
\hline Male & $43271(63.1)$ & $30817(69.2)$ & $4063(54.8)$ & $6755(63.3)$ & $1636(27.4)$ & \\
\hline Female & $25328(36.9)$ & 13736(30.8) & $3347(45.2)$ & $3908(36.7)$ & $4337(72.6)$ & \\
\hline Age & & & & & & $<0.001$ \\
\hline $18-49$ & $12230(17.8)$ & $7356(16.5)$ & $1236(16.7)$ & $3557(33.4)$ & $81(1.4)$ & \\
\hline $50-59$ & $16827(24.5)$ & $11062(24.8)$ & $2218(29.9)$ & $3115(29.1)$ & $432(7.2)$ & \\
\hline $60-69$ & $22019(32.1)$ & $15190(34.1)$ & $2632(35.5)$ & $2653(24.9)$ & $1544(25.8)$ & \\
\hline $70-79$ & $13322(19.4)$ & $8774(19.7)$ & $1113(15.1)$ & $1085(10.2)$ & $2350(39.3)$ & \\
\hline$\geq 80$ & $4201(6.2)$ & $2171(4.9)$ & $211(2.8)$ & $253(2.4)$ & $1566(26.3)$ & \\
\hline Race & & & & & & $<0.001$ \\
\hline White & $55394(80.8)$ & $37131(83.3)$ & $5842(78.8)$ & $7515(70.5)$ & $4906(82.1)$ & \\
\hline Black & $8030(11.7)$ & $3820(8.6)$ & $1168(15.8)$ & $2350(22.0)$ & 692(11.6) & \\
\hline Others $^{1}$ & $5175(7.5)$ & $3602(8.1)$ & $400(5.4)$ & $798(7.5)$ & $375(6.3)$ & \\
\hline Diagnosis year & & & & & & $<0.001$ \\
\hline $1973-1993$ & $15843(23.1)$ & $10490(23.5)$ & $1547(20.9)$ & 1890(17.7) & 1916(32.1) & \\
\hline 1994-2004 & $26157(38.1)$ & $17037(38.3)$ & 2894(39.1) & $4079(38.3)$ & $2147(35.9)$ & \\
\hline $2005-2015$ & $26599(38.8)$ & $17026(38.2)$ & $2969(40.0)$ & $4694(44.0)$ & $1910(32.0)$ & \\
\hline Pathological grade & & & & & & $<0.001$ \\
\hline Grade I & $8446(12.3)$ & $5368(12.0)$ & $893(12.1)$ & $1320(12.4)$ & $865(14.5)$ & \\
\hline Grade II & $34890(50.9)$ & $22638(50.8)$ & $3821(51.6)$ & $5385(50.5)$ & $3046(51.0)$ & \\
\hline Grade III & $20046(29.2)$ & 13191(29.6) & $2101(28.4)$ & $3154(29.6)$ & $1600(26.8)$ & \\
\hline Grade IV & $5217(7.6)$ & $3356(7.6)$ & $595(7.9)$ & $804(7.5)$ & $462(7.7)$ & \\
\hline AJCC stage & & & & & & $<0.001$ \\
\hline Stage I & $44110(64.3)$ & $28580(64.1)$ & $4803(64.8)$ & $6952(65.2)$ & $3775(63.2)$ & \\
\hline Stage II & $6822(9.9)$ & $4402(9.9)$ & $727(9.8)$ & $1129(10.6)$ & $564(9.4)$ & \\
\hline Stage III & $11165(16.3)$ & $7378(16.6)$ & $1141(15.4)$ & $1587(14.9)$ & $1059(17.8)$ & \\
\hline Stage IV & $6502(9.5)$ & $4193(9.4)$ & $739(10.0)$ & $995(9.3)$ & $575(9.6)$ & \\
\hline Tumor size & & & & & & $<0.001$ \\
\hline$\leq 5 \mathrm{~cm}$ & $37587(54.8)$ & $24464(54.9)$ & $4056(54.7)$ & $5836(54.7)$ & $3231(54.1)$ & \\
\hline $5-10 \mathrm{~cm}$ & $23197(33.8)$ & 15044(33.8) & $2533(34.2)$ & $3487(32.7)$ & 2133(35.7) & \\
\hline$>10 \mathrm{~cm}$ & $7815(11.4)$ & $5045(11.3)$ & $821(11.1)$ & $1340(12.6)$ & $609(10.2)$ & \\
\hline Surgery & & & & & & $<0.001$ \\
\hline Performed & $66507(96.9)$ & $43459(97.5)$ & $7131(96.2)$ & 10283(96.4) & $5634(94.3)$ & \\
\hline Not Performed & 2092(3.1) & $1094(2.5)$ & $279(3.8)$ & $380(3.6)$ & $339(5.7)$ & \\
\hline Median household income & & & & & & $<0.001$ \\
\hline Quartile 1 & $19671(28.7)$ & $12515(28.1)$ & $2314(31.2)$ & 2951(27.7) & 1891(31.7) & \\
\hline Quartile 2 & $15614(22.8)$ & $9958(22.4)$ & $1638(22.1)$ & $2634(24.7)$ & $1384(23.1)$ & \\
\hline Quartile 3 & $16913(24.7)$ & $11096(24.8)$ & $1859(25.1)$ & $2610(24.5)$ & $1348(22.6)$ & \\
\hline Quartile 4 & $16401(23.8)$ & $10984(24.7)$ & $1599(21.6)$ & $2468(23.1)$ & $1350(22.6)$ & \\
\hline \multicolumn{7}{|l|}{ Insurance status } \\
\hline Insured & $49926(72.8)$ & $32659(73.3)$ & $5532(74.7)$ & $7832(73.5)$ & $3903(65.3)$ & $<0.001$ \\
\hline Uninsured & $4836(7.1)$ & $2779(6.2)$ & $625(8.4)$ & $976(9.1)$ & $456(7.6)$ & \\
\hline Unknown & $13837(20.1)$ & $9115(20.5)$ & $1253(16.9)$ & $1855(17.4)$ & $1614(27.1)$ & \\
\hline
\end{tabular}

AJCC, the American Joint Committee on Cancer 
A

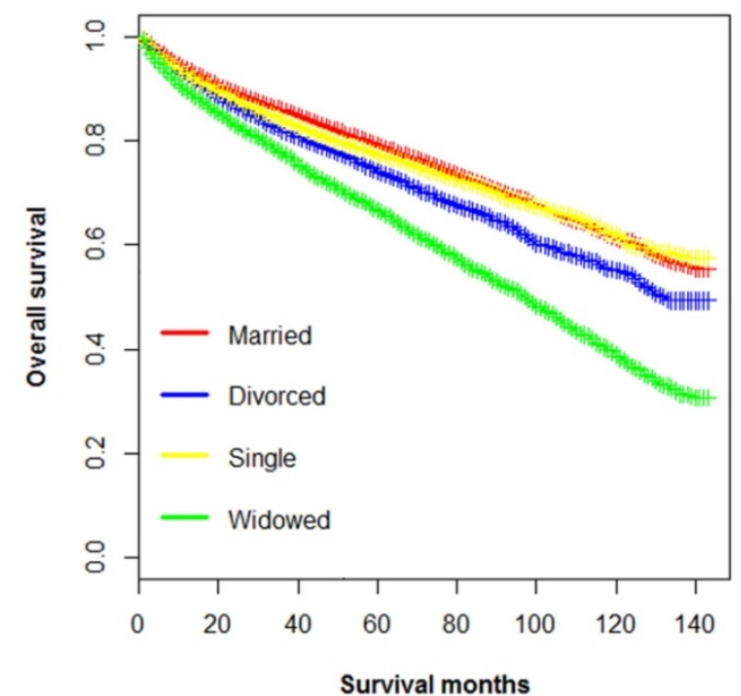

B

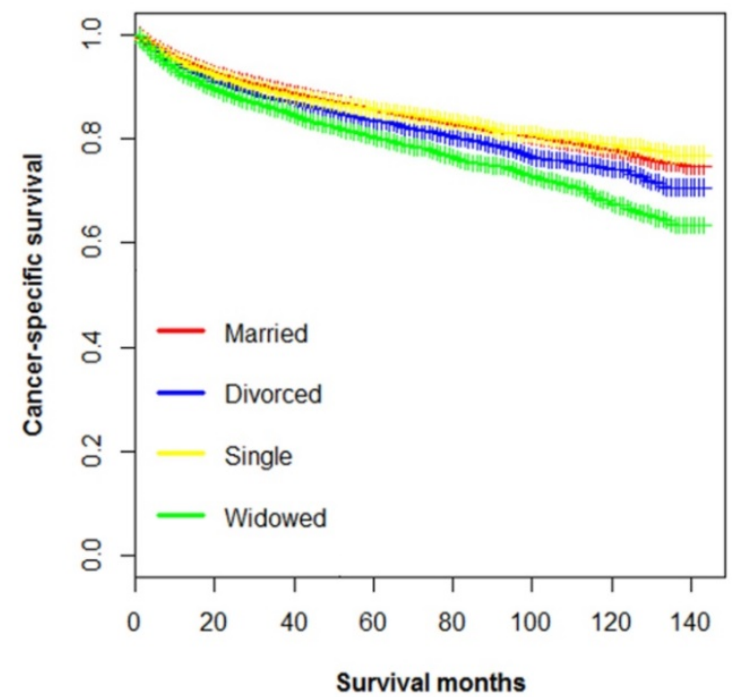

Figure 1. Kaplan-Meier survival curves according to marital status (married, divorced, widowed, and single) in patients with renal clear cell carcinoma. A. Overall survival: $X 2=$ $677, p<0.001$; B. Cancer-specific survival: $X 2=147, p<0.001$.

Using married patients as reference, unmarried individuals persisted to be associated with increased risks death for both OS (HR, 1.31, 95\% CI 1.24-1.38, $p<$ 0.001 ) and CSS (HR, 1.19, 95\% CI 1.11-1.28, $p<0.001)$. These results proved that our analysis was credible and reliable, which meant that the confounding factors were not responsible for the error source.

Table 2. Univariate and multivariate survival analysis for evaluating the impact of marital status on the OS among ccRCC patients

\begin{tabular}{|c|c|c|c|c|c|c|}
\hline \multirow[t]{2}{*}{ Variables } & \multirow{2}{*}{$\begin{array}{l}5 \text {-year } \\
\text { OS }\end{array}$} & \multicolumn{2}{|c|}{ Univariate analysis } & \multicolumn{3}{|c|}{ Multivariate analysis } \\
\hline & & Log Rank $\chi^{2}$ & $p$ & HR & $95 \% \mathrm{CI}$ & $p$ \\
\hline Marital status & & 677 & $<0.001$ & & & \\
\hline Married & $79.0 \%$ & & & Reference & & \\
\hline Divorced & $73.8 \%$ & & & 1.33 & $1.27-1.40$ & $<0.001$ \\
\hline Single & $77.3 \%$ & & & 1.26 & $1.20-1.32$ & $<0.001$ \\
\hline Widowed & $66.4 \%$ & & & 1.42 & $1.35-1.49$ & $<0.001$ \\
\hline Sex & & 95.8 & $<0.001$ & & & \\
\hline Male & $75.6 \%$ & & & Reference & & \\
\hline Female & $79.4 \%$ & & & 0.85 & $0.82-0.88$ & 0.021 \\
\hline Age & & 3156 & $<0.001$ & & & \\
\hline $18-49$ & $87.2 \%$ & & & Reference & & \\
\hline $50-59$ & $81.7 \%$ & & & 1.32 & $1.24-1.41$ & $<0.001$ \\
\hline $60-69$ & $77.1 \%$ & & & 1.71 & $1.61-1.82$ & $<0.001$ \\
\hline $70-79$ & $70.3 \%$ & & & 2.55 & $2.39-2.72$ & $<0.001$ \\
\hline$\geq 80$ & $54.2 \%$ & & & 4.01 & $3.73-4.32$ & $<0.001$ \\
\hline Race & & 33.1 & $<0.001$ & & & \\
\hline White & $76.8 \%$ & & & Reference & & \\
\hline Black & $76.7 \%$ & & & 1.14 & $1.08-1.19$ & $<0.001$ \\
\hline Others & $79.9 \%$ & & & 0.88 & $0.82-0.93$ & $<0.001$ \\
\hline Diagnosis year & & 173 & $<0.001$ & & & \\
\hline 1973-1993 & $73.6 \%$ & & & Reference & & \\
\hline 1994-2004 & $78.5 \%$ & & & 0.95 & $0.91-0.98$ & 0.043 \\
\hline $2005-2015$ & $82.5 \%$ & & & 0.87 & $0.82-0.92$ & $<0.001$ \\
\hline Pathological grade & & 6670 & $<0.001$ & & & \\
\hline Grade I & $85.1 \%$ & & & Reference & & \\
\hline Grade II & $84.4 \%$ & & & 0.97 & $0.93-1.04$ & 0.477 \\
\hline Grade III & $69.9 \%$ & & & 1.35 & $1.27-1.44$ & $<0.001$ \\
\hline Grade IV & $39.5 \%$ & & & 2.26 & $2.11-2.42$ & $<0.001$ \\
\hline Tumor size & & 5612 & $<0.001$ & & & \\
\hline$\leq 5 \mathrm{~cm}$ & $86.7 \%$ & & & Reference & & \\
\hline
\end{tabular}

\begin{tabular}{|c|c|c|c|c|c|c|}
\hline \multirow[t]{2}{*}{ Variables } & \multirow{2}{*}{$\begin{array}{l}\text { 5-year } \\
\text { OS }\end{array}$} & \multicolumn{2}{|c|}{ Univariate analysis } & \multicolumn{3}{|c|}{ Multivariate analysis } \\
\hline & & Log Rank $\chi^{2}$ & $p$ & HR & $95 \% \mathrm{CI}$ & $p$ \\
\hline $5-10 \mathrm{~cm}$ & $70.4 \%$ & & & 1.47 & $1.39-1.55$ & $<0.001$ \\
\hline$>10 \mathrm{~cm}$ & $50.6 \%$ & & & 1.67 & $1.55-1.79$ & $<0.001$ \\
\hline AJCC stage & & 26079 & $<0.001$ & & & \\
\hline Stage I & $87.5 \%$ & & & Reference & & \\
\hline Stage II & $80.9 \%$ & & & 1.07 & $1.01-1.14$ & $<0.001$ \\
\hline Stage III & $67.0 \%$ & & & 1.79 & $1.71-1.88$ & $<0.001$ \\
\hline Stage IV & $18.61 \%$ & & & 6.62 & $6.29-6.98$ & $<0.001$ \\
\hline Surgery & & 8574 & $<0.001$ & & & \\
\hline Performed & $78.70 \%$ & & & Reference & & \\
\hline Not Performed & $20.35 \%$ & & & 0.30 & $0.28-0.32$ & $<0.001$ \\
\hline $\begin{array}{l}\text { Median household } \\
\text { income }\end{array}$ & & 116 & $<0.001$ & & & \\
\hline Quartile 1 & $74.9 \%$ & & & & & \\
\hline Quartile 2 & $76.3 \%$ & & & 0.92 & $0.88-0.96$ & $<0.001$ \\
\hline Quartile 3 & $78.3 \%$ & & & 0.85 & $0.81-0.88$ & $<0.001$ \\
\hline Quartile 4 & $78.8 \%$ & & & 0.83 & $0.79-0.87$ & $<0.001$ \\
\hline Insurance status & & 72.5 & $<0.001$ & & & \\
\hline Insured & $78.1 \%$ & & & Reference & & \\
\hline Uninsured & $75.4 \%$ & & & 1.16 & $1.07-1.25$ & $<0.001$ \\
\hline
\end{tabular}

\section{Discussion}

To our best knowledge, this study is the first and largest study to date investigating the impact of marital status on the survival of ccRCC patients. In this large, population-based study, we firstly used a systematic PSM and subgroup analysis to show that marital status was an independent prognostic factor and contributed to worse OS and CSS for unmarried ccRCC patients. Compared to married patients, unmarried individuals, including divorced, single, and widowed, faced higher mortality risks for OS and CSS irrespective of whether or not the patients were adjusted for age, sex, race, diagnosis year, histological type, pathological grade, AJCC stage, tumor size, surgery condition, median household income or 
insurance status. Besides, among the unmarried, widowed patients had the best chance of dying from ccRCC even after adjustment the abovementioned variables. Moreover, in the subgroup analysis, the increased mortality risks were also significant among unmarried patients at each sex, surgery condition, and all cancer stages subgroups. Even in the 1:1 matched cohort with comparable baseline characteristics, unmarried status still contributed to worse survival for ccRCC patients.

Table 3. Univariate and multivariate survival analysis for evaluating the impact of marital status on the CSS among ccRCC patients

\begin{tabular}{|c|c|c|c|c|c|c|}
\hline \multirow[t]{2}{*}{ Variables } & \multirow{2}{*}{$\begin{array}{l}5 \text {-year } \\
\text { CSS }\end{array}$} & \multicolumn{2}{|c|}{ Univariate analysis } & \multicolumn{3}{|c|}{ Multivariate analysis } \\
\hline & & $\log \operatorname{Rank} \chi^{2}$ & $p$ & HR & $95 \% \mathrm{CI}$ & $p$ \\
\hline Marital status & & 147 & $<0.001$ & & & \\
\hline Married & $85.5 \%$ & & & Reference & & \\
\hline Divorced & $83.3 \%$ & & & 1.16 & $1.08-1.25$ & $<0.001$ \\
\hline Single & $80.5 \%$ & & & 1.09 & $1.02-1.16$ & 0.006 \\
\hline Widowed & $76.5 \%$ & & & 1.20 & $1.13-1.28$ & $<0.001$ \\
\hline Sex & & 5.3 & 0.021 & & & \\
\hline Male & $83.8 \%$ & & & Reference & & \\
\hline Female & $86.6 \%$ & & & 0.96 & $0.92-0.99$ & 0.011 \\
\hline Age & & 636 & $<0.001$ & & & \\
\hline $18-49$ & $90.0 \%$ & & & Reference & & \\
\hline $50-59$ & $86.5 \%$ & & & 1.14 & $1.05-1.23$ & $<0.001$ \\
\hline $60-69$ & $84.3 \%$ & & & 1.33 & $1.23-1.43$ & $<0.001$ \\
\hline $70-79$ & $82.3 \%$ & & & 1.67 & $1.54-1.80$ & $<0.001$ \\
\hline$\geq 80$ & $74.8 \%$ & & & 2.25 & $2.04-2.47$ & $<0.001$ \\
\hline Race & & 29.7 & $<0.001$ & & & \\
\hline White & $84.6 \%$ & & & Reference & & \\
\hline Black & $86.0 \%$ & & & 1.09 & $1.02-1.17$ & 0.011 \\
\hline Others $^{1}$ & $85.0 \%$ & & & 0.94 & $0.87-1.01$ & 0.102 \\
\hline Diagnosis year & & 21 & $<0.001$ & & & \\
\hline $1973-1993$ & $82.9 \%$ & & & Reference & & \\
\hline 1994-2004 & $85.5 \%$ & & & 0.94 & $0.89-1.00$ & 0.0431 \\
\hline $2005-2015$ & $87.7 \%$ & & & 0.84 & $0.79-0.90$ & $<0.001$ \\
\hline $\begin{array}{l}\text { Pathological } \\
\text { grade }\end{array}$ & & 2085 & $<0.001$ & & & \\
\hline Grade I & $94.2 \%$ & & & Reference & & \\
\hline Grade II & $92.4 \%$ & & & 1.09 & $0.99-1.20$ & 0.074 \\
\hline Grade III & $77.4 \%$ & & & 1.84 & $1.67-2.02$ & $<0.001$ \\
\hline Grade IV & $45.0 \%$ & & & 3.12 & $2.81-3.45$ & $<0.001$ \\
\hline Tumor size & & 613 & $<0.001$ & & & \\
\hline$\leq 5 \mathrm{~cm}$ & $95.0 \%$ & & & Reference & & \\
\hline $5-10 \mathrm{~cm}$ & $78.5 \%$ & & & 1.81 & $1.70-1.93$ & $<0.001$ \\
\hline$>10 \mathrm{~cm}$ & $55.2 \%$ & & & 2.25 & $2.09-2.42$ & $<0.001$ \\
\hline AJCC stage & & 5706 & $<0.001$ & & & \\
\hline Stage I & $95.5 \%$ & & & Reference & & \\
\hline Stage II & $87.8 \%$ & & & 1.59 & $1.45-1.74$ & $<0.001$ \\
\hline Stage III & $75.6 \%$ & & & 3.32 & $3.10-3.56$ & $<0.001$ \\
\hline Stage IV & $21.19 \%$ & & & 14.06 & $13.09-15.10$ & $<0.001$ \\
\hline Surgery & & 4883 & $<0.001$ & & & \\
\hline Performed & $86.5 \%$ & & & Reference & & \\
\hline Not Performed & $28.1 \%$ & & & 0.26 & $0.24-0.28$ & $<0.001$ \\
\hline $\begin{array}{l}\text { Median } \\
\text { household income }\end{array}$ & & 40.2 & $<0.001$ & & & \\
\hline Quartile 1 & $83.9 \%$ & & & Reference & & \\
\hline Quartile 2 & $84.1 \%$ & & & 0.96 & $0.91-1.02$ & 0.158 \\
\hline Quartile 3 & $85.3 \%$ & & & 0.88 & $0.83-0.93$ & $<0.001$ \\
\hline Quartile 4 & $85.7 \%$ & & & 0.87 & $0.82-0.92$ & $<0.001$ \\
\hline Insurance status & & 142 & $<0.001$ & & & \\
\hline Insured & $85.2 \%$ & & & Reference & & \\
\hline Uninsured & $83.2 \%$ & & & 1.04 & $0.96-1.14$ & 0.314 \\
\hline
\end{tabular}

AJCC, the American Joint Committee on Cancer; CSS, cancer-specific survival; CI, confidence interval; $\mathrm{HR}$, hazard ratio
Table 4. Univariate and multivariate analysis of marital status on the CSS among ccRCC patients according to age, surgery status, and cancer stages

\begin{tabular}{|c|c|c|c|c|c|c|}
\hline \multirow[t]{2}{*}{ Variables } & \multirow{2}{*}{$\begin{array}{l}5 \text {-year } \\
\text { CSS }\end{array}$} & \multicolumn{2}{|c|}{ Univariate analysis } & \multicolumn{3}{|c|}{ Multivariate analysis } \\
\hline & & $\begin{array}{l}\text { Log rank } \\
\chi^{2}\end{array}$ & $p$ & HR & $95 \%$ CI & $p$ \\
\hline \multicolumn{7}{|l|}{ Sex } \\
\hline Male & & 86.5 & $<0.001$ & & & \\
\hline Married & $84.5 \%$ & & & Reference & & \\
\hline Divorced & $80.3 \%$ & & & 1.12 & $1.04-1.21$ & $<0.001$ \\
\hline Single & $83.9 \%$ & & & 0.96 & 0.93-1.02 & 0.265 \\
\hline Widowed & $78.3 \%$ & & & 1.37 & $1.29-1.48$ & $<0.001$ \\
\hline Female & & 122 & $<0.001$ & & & \\
\hline Married & $87.8 \%$ & & & Reference & & \\
\hline Divorced & $86.9 \%$ & & & 1.26 & $1.16-1.37$ & $<0.001$ \\
\hline Single & $88.1 \%$ & & & 1.05 & $0.96-1.14$ & 0.247 \\
\hline Widowed & $81.6 \%$ & & & 2.12 & $1.99-2.26$ & $<0.001$ \\
\hline \multicolumn{7}{|l|}{ Surgery } \\
\hline Performed & & 35.6 & $<0.001$ & & & \\
\hline Married & $87.3 \%$ & & & Reference & & \\
\hline Divorced & $85.5 \%$ & & & 1.52 & $1.29-1.81$ & $<0.001$ \\
\hline Single & $86.9 \%$ & & & 1.49 & $0.85-2.65$ & 0.165 \\
\hline Widowed & $82.8 \%$ & & & 1.63 & $1.46-1.84$ & $<0.001$ \\
\hline $\begin{array}{l}\text { Not } \\
\text { performed }\end{array}$ & & 17.5 & $<0.001$ & & & \\
\hline Married & $32.2 \%$ & & & Reference & & \\
\hline Divorced & $26.8 \%$ & & & 1.16 & $0.98-1.36$ & 0.066 \\
\hline Single & $32.3 \%$ & & & 1.02 & $0.84-1.24$ & 0.834 \\
\hline Widowed & $24.2 .2 \%$ & & & 1.28 & $1.04-1.71$ & 0.015 \\
\hline \multicolumn{7}{|l|}{ AJCC stage } \\
\hline Stage I & & 145 & $<0.001$ & & & \\
\hline Married & $96.3 \%$ & & & Reference & & \\
\hline Divorced & $95.8 \%$ & & & 1.35 & $1.25-1.47$ & $<0.001$ \\
\hline Single & $96.2 \%$ & & & 1.09 & $1.02-1.19$ & $<0.001$ \\
\hline Widowed & $92.9 \%$ & & & 2.36 & $2.21-2.53$ & $<0.001$ \\
\hline Stage II & & 52.3 & $<0.001$ & & & \\
\hline Married & $88.9 \%$ & & & Reference & & \\
\hline Divorced & $84.2 \%$ & & & 1.51 & $1.29-1.77$ & $<0.001$ \\
\hline Single & $89.6 \%$ & & & 1.14 & $0.98-1.33$ & 0.088 \\
\hline Widowed & $79.6 \%$ & & & 2.12 & $1.83-2.47$ & $<0.001$ \\
\hline Stage III & & 22.9 & $<0.001$ & & & \\
\hline Married & $77.0 \%$ & & & Reference & & \\
\hline Divorced & $72.8 \%$ & & & 1.27 & $1.14-1.42$ & $<0.001$ \\
\hline Single & $75.2 \%$ & & & 1.09 & $0.99-1.21$ & 0.0784 \\
\hline Widowed & $69.1 \%$ & & & 1.73 & $1.57-1.91$ & $<0.001$ \\
\hline Stage IV & & 19.5 & $<0.001$ & & & \\
\hline Married & $22.5 \%$ & & & Reference & & \\
\hline Divorced & $18.9 \%$ & & & 1.11 & $1.02-1.22$ & 0.021 \\
\hline Single & $19.0 \%$ & & & 1.14 & $1.05-1.23$ & 0.002 \\
\hline Widowed & $18.3 \%$ & & & 1.26 & $1.14-1.39$ & $<0.001$ \\
\hline
\end{tabular}

More importantly, our study identified several additional associations. Like, ccRCC tended to occur in young adults, more male than female, and male ccRCC patients benefited more from marriage than females. A potential reason for this sex disparity is that male patients might receive more social supports and assistance from their relatives or friends ${ }^{15}$. Interestingly, studies have suggested that the socioeconomic status is associated with better prognosis in several cancers ${ }^{16-18}$. In our study, this trend was also observed, and the widowed group had the lowest percentage of insurance, which may partly lead to their survival disadvantages. Additionally, it has been pointed that delayed diagnosis could 
contribute to poor survival among unmarried patients 8,19 . However, in our study, incidence of early stage (stage I/II) cancer was higher in the single group $(65.2 \%)$, divorced $(64.8 \%)$, and widowed $(63.2 \%)$ groups, compared with the married $(64.1 \%)$. Though, the SEER database does not provide information regarding cancer screening results, earlier diagnosis for unmarried people may indicate higher odds for cancer screening. Obviously, for ccRCC patients, delayed diagnosis alone couldn't explain the worse survival.

Our results indicated that unmarried patients experienced a significant survival disadvantage over their unmarried counterparts. Despite unclear

\section{A}

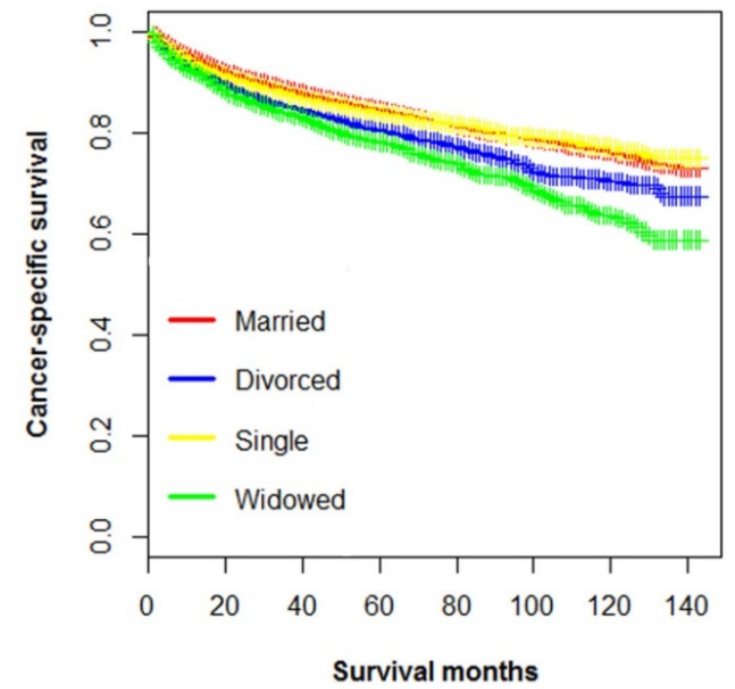

mechanisms intrinsic mechanisms behind this association, we try to analyze several possible reasons. First of all, people in good marital condition may be encouraged by their spouse to regular physical check-up. Moreover, married patients usually has better tolerance and adherence to prescribed treatments due to the medication reminders and assistance for their spouse ${ }^{20}$, which is crucial to improve the curability rate and survival ${ }^{21-23}$. Secondly, married patients usually possess stronger financial resources such as higher income level, better employment, medical insurance, which ultimately affect the access to early prevention, timely diagnosis and treatment ${ }^{24}$.

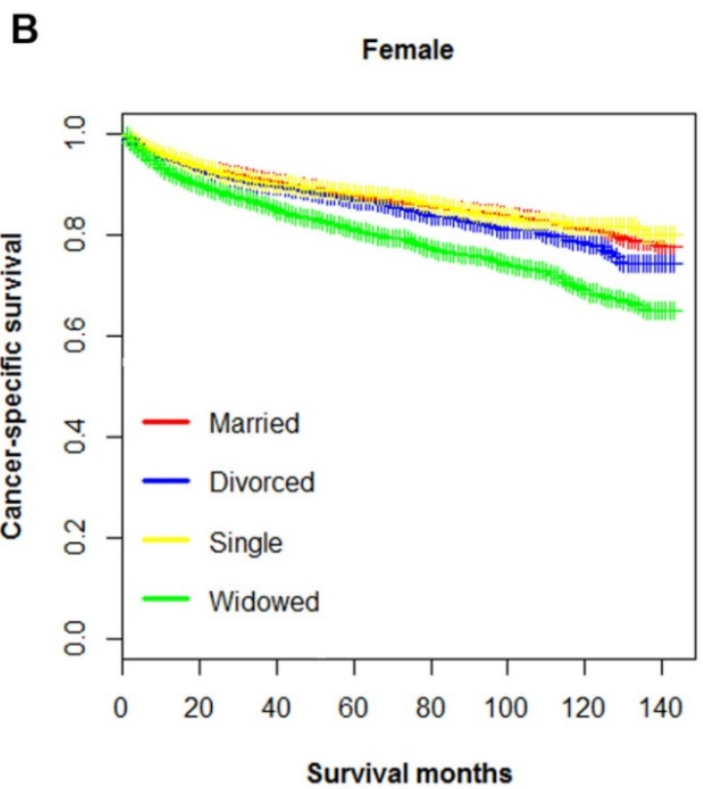

Figure 2. Kaplan-Meier survival curves of cancer-specific survival in patients with renal clear cell carcinoma stratified by sex. A. male: $X 2=90.9, p<0.001 ;$ B. female: $X 2=148$, $p<0.001$.

A

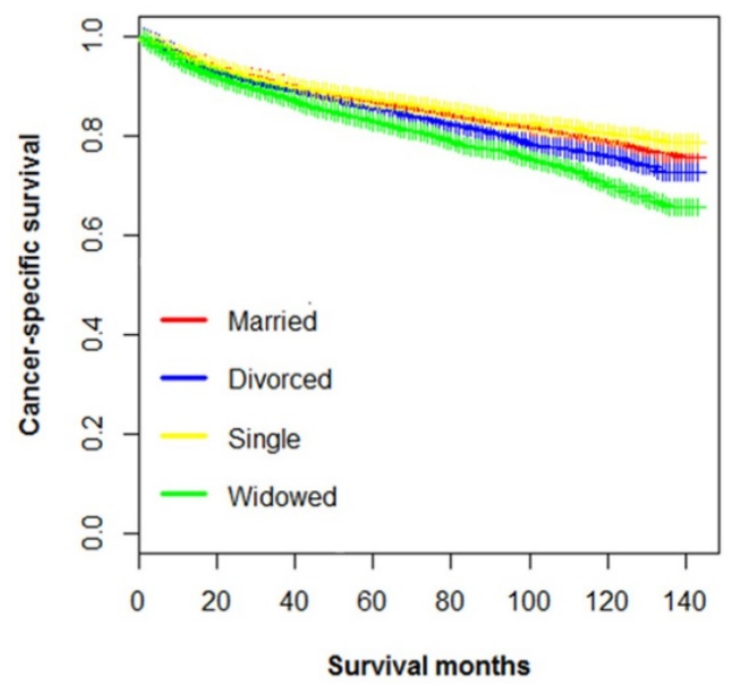

B

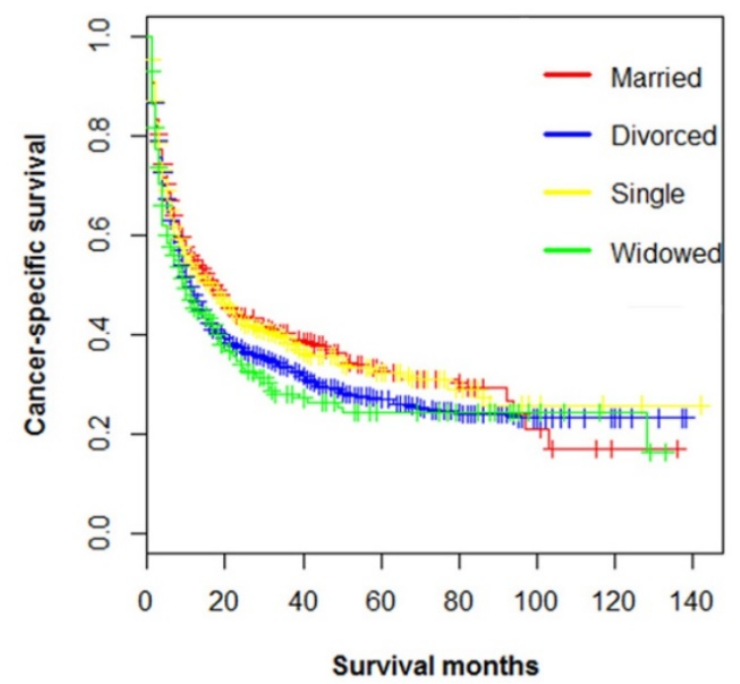

Figure 3. Kaplan-Meier survival curves of cancer-specific survival in patients with renal clear cell carcinoma stratified by surgery. A. surgery performed: $X 2=95.4, p<0.001$; B. surgery not performed: $\mathrm{X} 2=8.5, p=0.040$. 

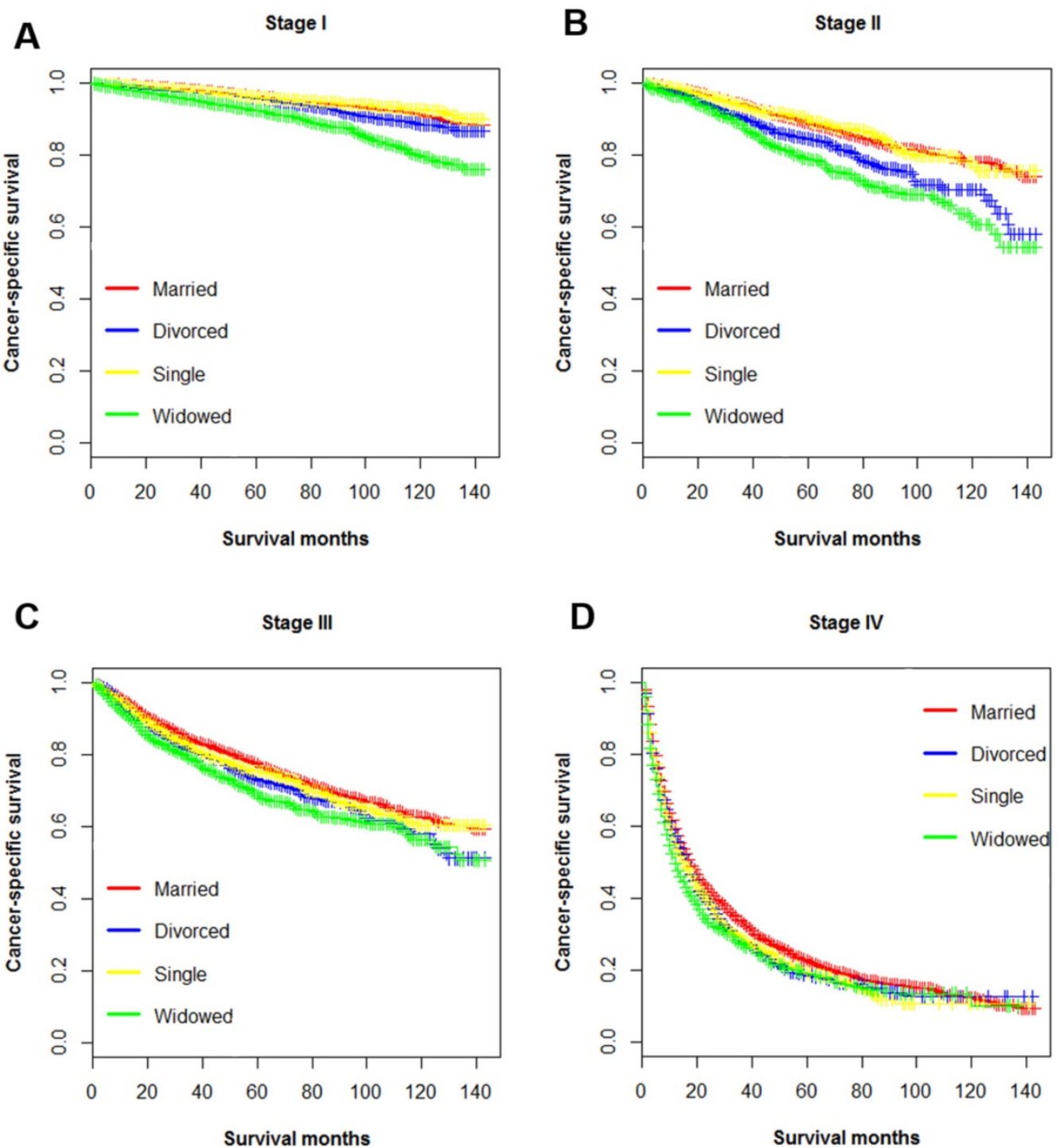

Figure 4. Kaplan-Meier survival curves of cancer-specific survival in patients with renal clear cell carcinoma stratified by tumor stage at diagnosis. A. stage $\mathrm{I}: X 2=186, p<0.001$; B. stage II: $X 2=57.8, p<0.001 ;$ C. stage III: $X 2=27.2, p<0.001 ;$ D. stage IV: $X 2=19.8, p<0.001$

A

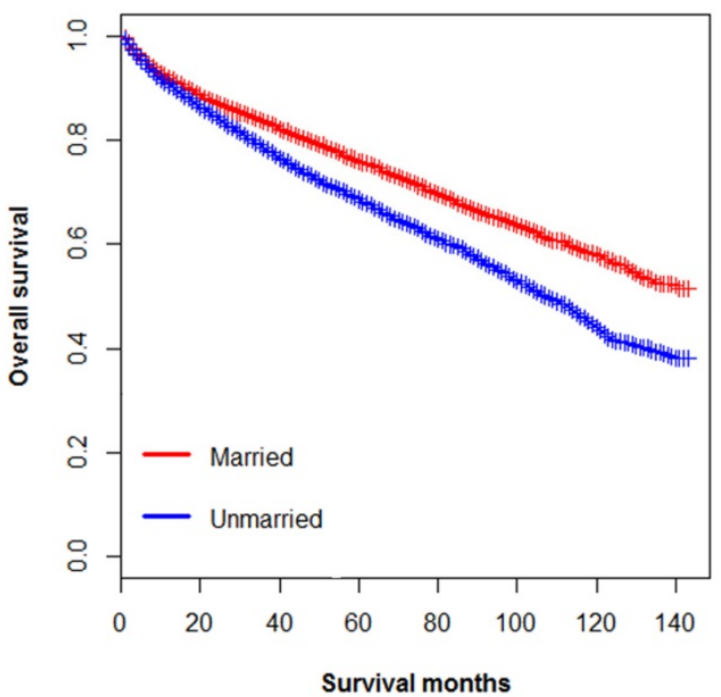

B

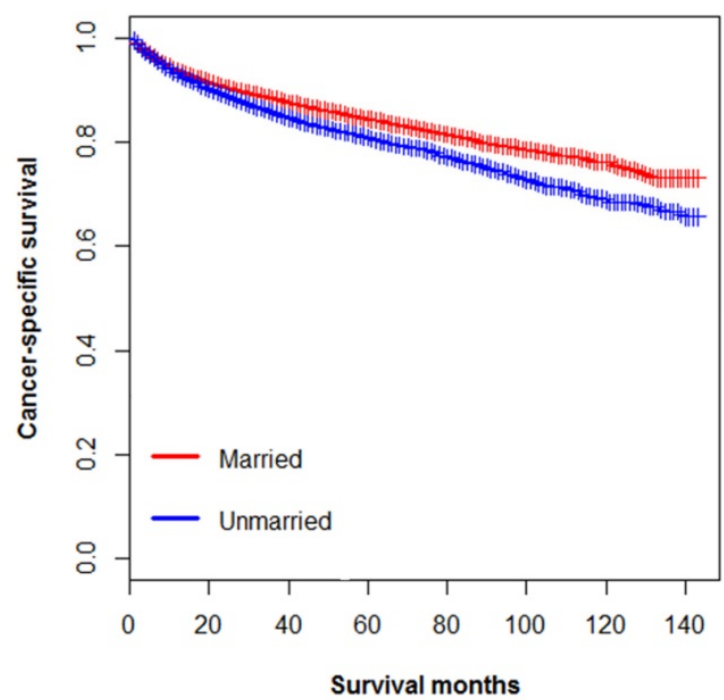

Figure 5. Kaplan-Meier survival curves according to marital status (married, unmarried) in patients with renal clear cell carcinoma. A. Overall survival: $X 2=121.4, p<0.001$; B. Cancer-specific survival: $\mathrm{X} 2=66.42, p<0.001$. 
Table 5. Patient baseline characteristics before and after PSM

\begin{tabular}{|c|c|c|c|c|c|c|c|c|}
\hline \multirow[t]{2}{*}{ Characteristic } & \multicolumn{4}{|l|}{ Before matching } & \multicolumn{4}{|c|}{ After matching } \\
\hline & Married (\%) & Unmarried (\%) & SD & $p^{*}$ & Married (\%) & Unmarried (\%) & SD & $p^{*}$ \\
\hline Sample size & 44553(100) & $24046(100)$ & & & 9014(100) & 9014(100) & & \\
\hline Sex & & & & $<0.001$ & & & & 0.004 \\
\hline Male & $30817(69.17)$ & $12454(51.79)$ & NA & & $4805(53.3)$ & $4611(52.2)$ & NA & \\
\hline Female & 13736(30.83) & $11592(48.21)$ & 0.46 & & $4209(46.7)$ & $4403(48.8)$ & 0.02 & \\
\hline Age & & & & $<0.001$ & & & & $<0.001$ \\
\hline $18-49$ & $7356(16.5)$ & $4874(20.3)$ & NA & & $88(1.0)$ & $81(0.9)$ & NA & \\
\hline $50-59$ & $11062(24.8)$ & $5765(24.0)$ & 0.43 & & $2336(25.9)$ & $2463(27.3)$ & 0.01 & \\
\hline $60-69$ & $15190(34.1)$ & $6829(28.4)$ & 0.47 & & $4157(46.1)$ & $3847(42.7)$ & -0.03 & \\
\hline $70-79$ & 8774(19.7) & $4548(18.9)$ & 0.40 & & $1102(12.2)$ & $1085(12.0)$ & 0.00 & \\
\hline$\geq 80$ & $2171(4.9)$ & $2030(8.4)$ & 0.22 & & 1331(14.8) & $1538(17.1)$ & 0.02 & \\
\hline Race & & & & $<0.001$ & & & & 0.561 \\
\hline White & $37131(83.3)$ & $18263(76.0)$ & NA & & $6673(74.0)$ & $6704(74.4)$ & NA & \\
\hline Black & $3820(8.6)$ & $4210(17.5)$ & 0.28 & & 1733(19.2) & 1682(18.7) & -0.01 & \\
\hline Others & $3602(8.1)$ & $1573(6.5)$ & 0.27 & & $608(6.7)$ & $628(7.0)$ & 0.00 & \\
\hline Diagnosis year & & & & $<0.001$ & & & 0.01 & 0.964 \\
\hline $1973-1993$ & $10490(23.5)$ & $5353(22.3)$ & NA & & $10490(23.5)$ & $5353(22.3)$ & NA & \\
\hline $1994-2004$ & $17037(38.2)$ & $9120(37.9)$ & 0.49 & & $17037(38.2)$ & $9120(37.9)$ & 0.00 & \\
\hline $2005-2015$ & $17026(38.2)$ & $9573(39.8)$ & 0.49 & & $17026(38.2)$ & $9573(39.8)$ & 0.00 & \\
\hline Pathological grade & & & & 0.002 & & & -0.01 & 0.267 \\
\hline Grade I & $5368(12.0)$ & $3078(12.8)$ & NA & & $1086(12.0)$ & 1093(12.1) & NA & \\
\hline Grade II & $22638(50.8)$ & $12252(51.0)$ & 0.50 & & $4568(50.7)$ & $4509(50.0)$ & -0.01 & \\
\hline Grade III & 13191(29.6) & $6855(28.5)$ & 0.46 & & $2753(30.5)$ & $2738(30.4)$ & 0.00 & \\
\hline Grade IV & $3356(7.5)$ & $1861(7.7)$ & 0.26 & & $607(6.7)$ & $674(7.5)$ & 0.01 & \\
\hline AJCC stage & & & & & & & & 0.495 \\
\hline Stage I & $28580(64.1)$ & $15530(64.6)$ & 0.33 & 0.048 & $5769(64.0)$ & $5680(63.0)$ & 0.00 & \\
\hline Stage II & $4402(9.9)$ & $2420(10.1)$ & 0.30 & & $883(9.8)$ & $886(9.8)$ & 0.00 & \\
\hline Stage III & $7378(16.6)$ & $3787(15.7)$ & 0.37 & & $1521(16.9)$ & $1563(17.3)$ & 0.00 & \\
\hline Stage IV & 4193(9.4) & $2309(9.6)$ & 0.29 & & $841(9.3)$ & $885(9.8)$ & 0.00 & \\
\hline Tumor size & & & & 0.626 & & & 0.01 & 0.196 \\
\hline$\leq 10 \mathrm{~cm}$ & $24464(54.9)$ & $13123(54.6)$ & NA & & $4890(54.2)$ & $4783(53.1)$ & NA & \\
\hline $10-20 \mathrm{~cm}$ & $15044(33.8)$ & $8153(33.9)$ & 0.47 & & $3165(35.1)$ & $3214(35.7)$ & 0.01 & \\
\hline$>20 \mathrm{~cm}$ & $5045(11.3)$ & $2770(11.5)$ & 0.32 & & $959(10.6)$ & 1017(11.3) & 0.01 & \\
\hline Surgery & & & & $<0.001$ & & & & 0.601 \\
\hline Performed & $43459(97.54)$ & 23048(95.85) & 0.18 & & $8589(95.1)$ & $8574(95.1)$ & 0.00 & \\
\hline Not Performed & $1094(2.46)$ & $998(4.15)$ & 0.15 & & $425(4.9)$ & 4404.9) & 0.00 & \\
\hline Median household income & & & & $<0.001$ & & & & 0.723 \\
\hline Quartile 1 & $12515(28.1)$ & $7156(29.8)$ & NA & & 2984(33.1) & $2960(32.8)$ & NA & \\
\hline Quartile 2 & $9958(22.4)$ & $5656(23.5)$ & 0.42 & & $2370(26.3)$ & $2405(26.7)$ & 0.00 & \\
\hline Quartile 3 & $11096(24.9)$ & $5817(24.2)$ & 0.43 & & 1905(21.1) & 1943(21.6) & -0.01 & \\
\hline Quartile 4 & $10984(24.7)$ & $5417(22.5)$ & 0.43 & & 1755(19.5) & $1706(18.9)$ & -0.01 & \\
\hline Insurance status & & & & $<0.001$ & & & & 0.638 \\
\hline Insured & $32659(73.3)$ & $17267(71.8)$ & NA & & 6374(70.7) & $6430(71.3)$ & NA & \\
\hline Uninsured & $2779(6.2)$ & $2057(8.6)$ & 0.24 & & $887(9.8)$ & $876(9.7)$ & 0.00 & \\
\hline Unknown & $9115(20.5)$ & $4722(19.6)$ & 0.40 & & $1753(19.4)$ & $1708(18.9)$ & 0.00 & \\
\hline
\end{tabular}

AJCC, the American Joint Committee on Cancer; SD, standardized difference; NA, not applicable. ${ }^{*} p$ value for $\chi 2$ test.

Thirdly, emotional support from spouse and other family members also contributed to better prognosis 25 compared to unmarried counterparts, unmarried patients, especially the widowed, have to face with higher psychological stress, economic pressures, lack of family support, absence of public assistance policy, leading to psychological imbalance, feel distressful and depressed. Multiple studies have confirmed that depression and stress were strongly associated with carcinoma growth and metastasis ${ }^{26-28}$. In addition, excess stress and depression would dysregulate the immune and endocrine function, induce chronic inflammation and thus result in worse survival29-31.

In our study, approximately $73 \%$ widowed patients were female. Miller et.al have reported that natural killer cells decreased significantly in women whose husbands have died recently ${ }^{28}$. And significantly higher proportion of psychological disturbance have been found in widowed patients. It is rather intuitive that lack of social support could seriously damage the function of immune cells, leading immune escape in tumor cells. On the other hand, it has been proposed that widowed patients less tended to receive surgery compared with the married. And undertreatment could have been one explanation for poor survival in widowed patients ${ }^{22}$. This correlation between marital status and receipt of surgery was independently validated in ccRCC patients in our study. Spouses of these married individuals might encourage them to receive surgery rather than conservative treatment, which could in part account for survival discrepancies.

Our study used a large sample size and 
sophisticated statistical analysis to investigate in depth the impact of marital status on survival for ccRCC patients, to ensure reliability. However, some potential limitations cannot be ignored. Firstly, we just simply divided patients into the married and unmarried groups but failed to get more details about their marriage. For example, SEER database do not provide information about marital history, family problems, lifestyle factors, which may serve as potential confounding factors. Secondly, the marital status was recorded only when diagnosed and we might put some patients into the wrong group when their marital status had changed during the follow-up. In addition, for unrecorded marital status (gay, lesbian, bisexual and transgender), we can't rule out that some have regular partner and get social support secretly. Thirdly, as far as the whole population concerned, a predominance of white people was observed in our study. Several studies have showed that racial disparities could lead to various prognoses among ccRCC patients ${ }^{32-34}$. One final but important point, SEER database does not provide several important clinical information regarding the treatment plan, co-morbid diseases and prognostic biomarkers such as VHL, HIF- $\alpha$ and ALDH2, which had proven to have an effect on RCC patient prognosis ${ }^{35}$.

\section{Conclusion}

Marital status was an independent prognostic factor of survival for ccRCC patients. Unmarried patients faced higher mortality risks for overall and cancer-specific survival, and among these patients, the widowed suffered the highest mortality risks. Unfavorable socioeconomic and psychological status might be responsible for the inferior survival of unmarried patients.

\section{Acknowledgements}

The present study was supported by grants from National Natural Science Foundation of China (NO. 81872245 and 81803601).

\section{Competing Interests}

The authors have declared that no competing interest exists.

\section{References}

1. Znaor A, Lortet-Tieulent J, Laversanne M, et al. International variations and trends in renal cell carcinoma incidence and mortality. Eur Urol. 2015;67:519-30.

2. Siegel RL, Miller KD, Jemal A. Cancer Statistics, 2017. CA Cancer J Clin. 2017;67:7-30.

3. Engel GL. The need for a new medical model: a challenge for biomedicine. Psychodyn Psychiatry. 2012;40:377-96.

4. Adler RH. Engel's biopsychosocial model is still relevant today. J Psychosom Res. 2009;67:607-11.
5. Aizer AA, Chen MH, McCarthy EP, et al. Marital status and survival in patients with cancer. J Clin Oncol. 2013;31:3869-76.

6. Zhang SL, Wang WR, Liu ZJ, et al. Marital status and survival in patients with soft tissue sarcoma: A population-based, propensity-matched study. Cancer Med. 2019;8:465-79.

7. Wu C, Chen P, Qian JJ, et al. Effect of marital status on the survival of patients with hepatocellular carcinoma treated with surgical resection: an analysis of 13,408 patients in the surveillance, epidemiology, and end results (SEER) database. Oncotarget. 2016;7:79442-52.

8. Liu Q, Luo D, Cai S, et al. Real-World Implications of Nonbiological Factors with Staging, Prognosis and Clinical Management in Colon Cancer. Cancers. 2018;10:263.

9. Li Y, Zhu MX, Qi SH. Marital status and survival in patients with renal cell carcinoma. Medicine. 2018;97:e0385.

10. Miao T, Li Y, Sheng X, et al. Marital status and survival of patients with kidney cancer. Oncotarget. 2017;8:86157-67.

11. Wang H, Wang L, Kabirov I, et al. Impact of marital status on renal cancer patient survival. Oncotarget. 2017;8:70204-13.

12. Warren JL, Klabunde CN, Schrag D, et al. Overview of the SEER-Medicare data: content, research applications, and generalizability to the United States elderly population. Med Care. 2002;40:Iv-3-18.

13. Austin PC. The use of propensity score methods with survival or time-to-event outcomes: reporting measures of effect similar to those used in randomized experiments. Stat Med. 2014;33:1242-58.

14. Austin PC. Balance diagnostics for comparing the distribution of baseline covariates between treatment groups in propensity-score matched samples. Stat Med. 2009;28:3083-107.

15. Merluzzi J, Dobrev SD. Unequal on top: Gender profiling and the income gap among high earner male and female professionals. Soc Sci Res. 2015;53:45-58.

16. Mahal A, Mahal B, Nguyen P, et al. Prostate cancer outcomes for men aged younger than 65 years with Medicaid versus private insurance. Cancer. 2018;124:752-9.

17. Rong X, Yang W, Garzon-Muvdi T, et al. Influence of insurance status on survival of adults with glioblastoma multiforme: A population-based study. Cancer. 2016;122:3157-65.

18. Ellis L, Canchola A, Spiegel D, et al. Trends in Cancer Survival by Health Insurance Status in California From 1997 to 2014. JAMA oncology. 2018;4:317-23.

19. Lin D, Gold HT, Schreiber D, et al. Impact of socioeconomic status on survival for patients with anal cancer. Cancer. 2018;124:1791-7.

20. Haley WE. Family caregivers of elderly patients with cancer: understanding and minimizing the burden of care. J Support Oncol. 2003;1:25-9.

21. Tammemagi CM, Neslund-Dudas C, Simoff M, et al. Lung carcinoma symptoms--an independent predictor of survival and an important mediator of African-American disparity in survival. Cancer. 2004;101:1655-63.

22. Ferrante JM, Gonzalez EC, Roetzheim RG, et al. Clinical and demographic predictors of late-stage cervical cancer. Arch Fam Med. 2000;9:439-45.

23. Nayeri K, Pitaro G, Feldman JG. Marital status and stage at diagnosis in cancer. N Y State J Med. 1992;92:8-11.

24. Moreno-Smith M, Lutgendorf SK, Sood AK. Impact of stress on cancer metastasis. Future Oncol. 2010;6:1863-81.

25. Wittmann D. Emotional and sexual health in cancer: partner and relationship issues. Curr Opin Support Palliat Care. 2016;10:75-80.

26. Janssen LME, Ramsay EE, Logsdon CD, et al. The immune system in cancer metastasis: friend or foe? J Immunother Cancer. 2017;5:79.

27. Sullivan DR, Forsberg CW, Ganzini L, et al. Depression symptom trends and health domains among lung cancer patients in the CanCORS study. Lung Cancer. 2016;100:102-9.

28. Miller AH, Raison CL. The role of inflammation in depression: from evolutionary imperative to modern treatment target. Nat Rev Immunol. 2016;16:22-34.

29. Uchino BN. Social support and health: a review of physiological processes potentially underlying links to disease outcomes. J Behav Med. 2006;29:377-87.

30. Sapolsky RM, Donnelly TM. Vulnerability to stress-induced tumor growth increases with age in rats: role of glucocorticoids. Endocrinology. 1985;117:662-6.

31. Miller GE, Cohen S, Ritchey AK. Chronic psychological stress and the regulation of pro-inflammatory cytokines: a glucocorticoid-resistance model. Health Psychol. 2002;21:531-41.

32. Patel HD, Kates M, Pierorazio PM, et al. Race and sex disparities in the treatment of older patients with T1a renal cell carcinoma: a comorbidity-controlled competing-risks model. Urol Oncol. 2014;32:576-83. 
33. Mafolasire A, Yao X, Nawaf C, et al. Racial disparities in renal cell carcinoma: a single-payer healthcare experience. Cancer Med. 2016;5:2101-8.

34. Kates M, Whalen MJ, Badalato GM, et al. The effect of race and gender on the surgical management of the small renal mass. Urol Oncol. 2013;31:1794-9.

35. Gao Y, Wu Z, Xie L, et al. VHL deficiency augments anthracycline sensitivity of clear cell renal cell carcinomas by down-regulating ALDH2. Nat Commun. 2017;8:15337. 